\title{
Sparganosis - neglected zoonosis and its reservoir in wildlife ${ }^{11}$
}

\author{
MARTA KOKODZIEJ-SOBOCIŃSKA, MARIUSZ MINIUK*
}

\begin{abstract}
Mammal Research Institute, Polish Academy of Sciences, Waszkiewicza 1, 17-230 Białowieża, Poland *Veterinary office, Ogrodowa 4, 17-230 Białowieża, Poland
\end{abstract}

Kołodziej-Sobocińska M., Miniuk M.

\section{Sparganosis - neglected zoonosis and its reservoir in wildlife}

\section{Summary}

Emerging parasitic invasions may have a significant influence on wild and domestic animals, as well as humans worldwide. One of the possible sources of infection is the consumption of the meat of wild animals that enable infectious agents to circulate in the environment and transmit them to human and domesticated animal hosts. Human sparganosis is an emerging and neglected zoonosis worldwide. Most cases are known from Asia; however, a few cases have also been diagnosed in Europeans. Recently, in Białowieża Primeval Forest, northeastern Poland, cases of sparganosis were stated in both intermediate (raccoon dog, badger, wild boar) and definitive hosts (wolf, lynx). Confirmed presence of sparganosis in game species (wild boar) and the possibility of its occurrence in domestic animals (dogs and cats) must be taken into consideration in case this zoonosis is not routinely diagnosed by veterinarians. Plerocercoids in wild boar are located subcutaneously and in muscle tissue. Thus, the emergence of human sparganosis due to consumption of undercooked or smoked wild boar meat is very likely in the areas where wild boar is an approved food source. In this review we describe the biology of Spirometra sp. and the current knowledge about the spread of this tapeworm among wild animals as well as the risk for people, focusing on the situation in Europe.

Keywords: Spirometra sp., sparganosis, wild animals, Poland

Emerging parasitic invasions in wildlife may have a significant influence on individuals and populations (15). There are many environmental and biological factors involved in the parasitism: climate conditions, behavioral traits (e.g. host sociality), population density, age, diet (e.g. prey abundance), habitat, age, sex, host immunocompetence and others $(5,10,23,28$, 29). However, the spread of parasites in populations of vertebrates and factors affecting prevalence and intensity of parasitosis in wildlife are not often studied. Moreover, parasitic invasions in wildlife may have a significant influence on domestic animals and humans worldwide. One of the possible sources of infection is consumption of the meat of wild animals that enable infectious agents to circulate in the environment and transmit them to human and domesticated animal hosts. Spirometra sp. tapeworm is one of the neglected parasitic agents with zoonotic potential.

1) The study was financed by the National Science Centre, project No. 2016/21/B/NZ8/02429.

\section{Spirometra life cycle and occurrence in Europe}

Sparganosis is a parasitic disease caused by plerocercoid larvae of Spirometra sp. (Diphyllobothridae). In wildlife, the cestode reproduces in cat- and doglike carnivores such as lynxes (Lynx lynx) and wolves (Canis lupus) (6). The first intermediate hosts are copepods, where procercoids infective for the second intermediate hosts develop. The second intermediate hosts can be many vertebrate species from the amphibians, reptiles, birds or mammals in which plerocercoids causing sparganosis develop $(9,26)$. Several animal species that are not definitive hosts serve as paratenic or transport hosts. In this case, the larvae which are acquired by feeding on animals infected with plerocercoids encyst once more after passing through the intestinal wall and migrating to tissues (Fig. 1). The most common localizations of the sparganum are subcutaneous connective tissue (Fig. 2) $(9,27)$. However, they can also be found in muscles (Fig. 3) (8). 


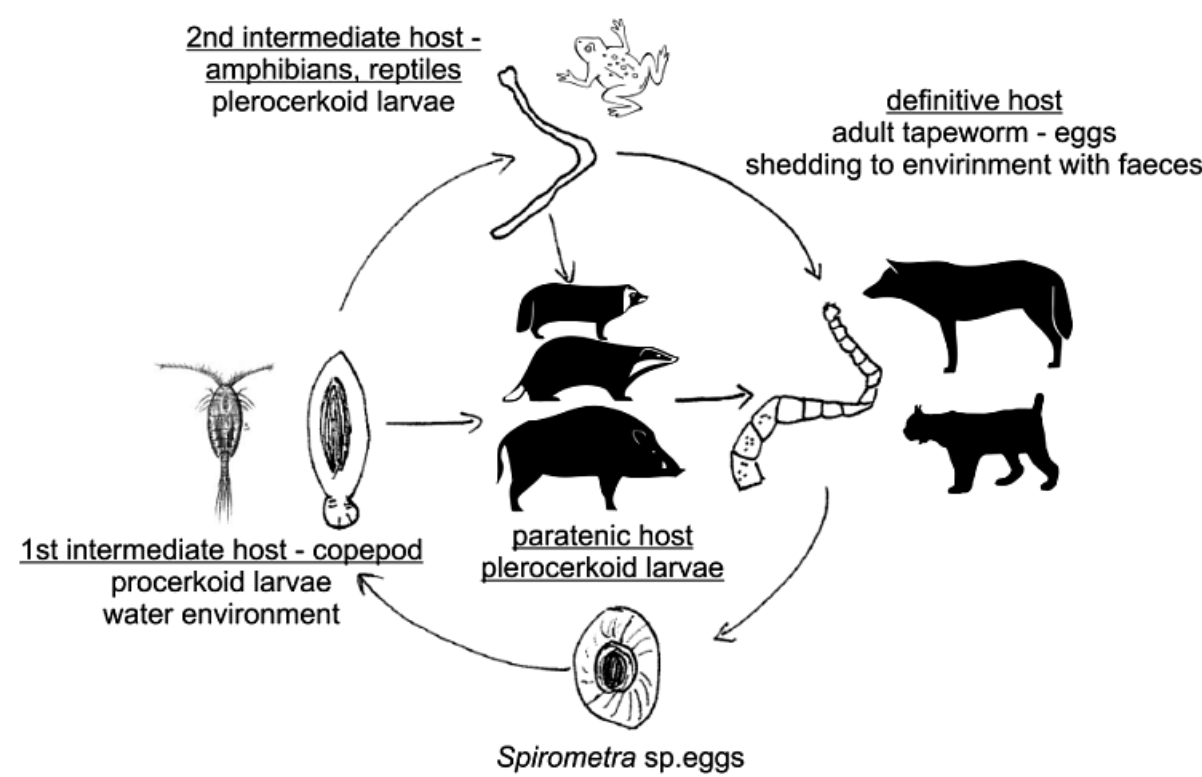

Fig. 1. Life cycle of Spirometra sp. Illustrated by M. Kołodziej-Sobocińska
Sparganosis was first described by Manson in 1882 (14). Most of the research on sparganosis was conducted in Asia, where sparganosis is a serious problem to humans. Several species of medical interest have been described: Spirometra mansoni, $S$. mansonoides, $S$. ranarum, $S$. erinaceieuropaei, $S$. theileri and $S$. proliferum (27).

In wildlife, sparganosis had been previously recorded in the European hedgehog (Erinaceus europeaus), stone marten (Martes foina), polecat (Mustela putorius), brown rat (Mus decumanus), edible frog (Rana esculenta), ringed snake (Natrix torquata), viperine snake (Tropidonotus viperinus), grass snake (Tropidonotus natrix) in Italy (17), as well as in the European mink (Mustela lutreola), American mink (Neovison vison), river otter (Lutra lutra), raccoon dog (Nyctereutes procyonoides), stoat (Mustela erminea) and weasel (Mustela nivalis) in Belarus (1, 19-22). In Poland, only one species of Spirometra (S. janickii) has been described as a new species by Furmaga (6). It was found in lynx (Lynx lynx) and wolf (Canis lupus) from the Białowieża Primeval Forest (BPF). These large carnivores are definitive hosts for Spirometra (6, 24). Plerocercoids of this tapeworm were also found in: shrew (Sorex araneus), red fox (Vulpes vulpes) (6), badger (Meles meles) (9), wild boar (Sus scrofa) (8), raccoon dog and river otter (Kołodziej-Sobocińska unpublished data) - all in BPF.

\section{Documented cases of sparganosis in Polish wildife}

Badger. In April 2013, an adult female Eurasian badger, killed by a car on the road in BPF was found (9). At necropsy, 128 complete and 40 fragments (damaged during extraction) of spargana of the tapeworm Spirometra sp. were isolated. They were located subcutaneously, mainly on the hind legs and along the spine (Fig. 2). Mean length of spargana was $87 \pm 38 \mathrm{~mm}$ (range: $28-213 \mathrm{~mm}$ ). Subcutaneous thickenings (cysts) were numerous on the whole carcass. No adult Spirometra sp. tapeworms were found in the animal intestines. Over $500 \mathrm{bp}$ length sequence of an evolutionary conserved nuclear 18S rRNA gene was used for genetic testing of the species, according to the procedure described in detail by Lee et al. (12). The sequences obtained from plerocercoids isolated from badger were published by Kołodziej-Sobocińska et al. (9). They were compared with GenBank deposits using nucleotide BLAST search and showed 99\% identity with Spirometra erinaceieuropaei (9). Thus the investigation confirmed the recognition of the recovered spargana as Spirometra erinaceieuropaei.
Fig. 3. Plerocercoid larvae of Spirometra sp. isolated from muscle tissue of wild boar hunted in Białowieża Primeval Forest, Poland (photo by M. Kołodziej-Sobocińska) 


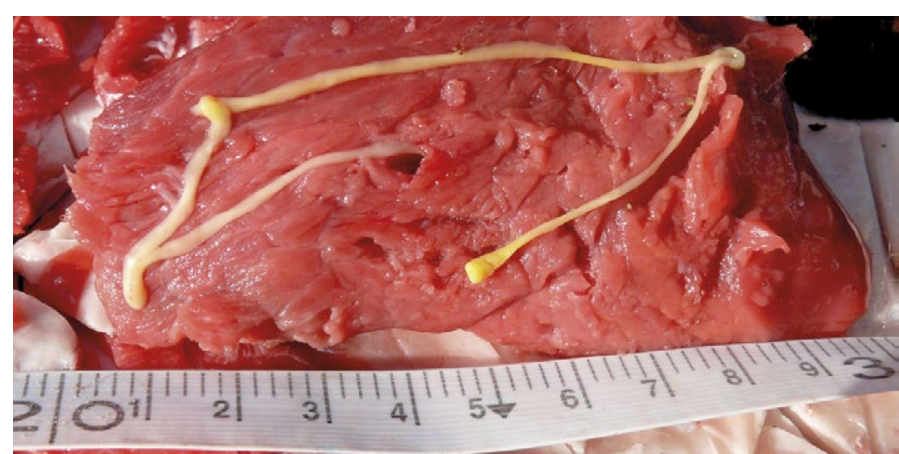

Fig. 4. Plerocercoid larvae of Spirometra sp. isolated from muscle tissue of wild boar hunted in Białowieża Primeval Forest, Poland. The average length of sparganum was $103 \mathrm{~mm}$ (range 30-290 mm) (photo by M. Kołodziej-Sobocińska)

Wild boar. In February and March 2016 plerocercoids of Spirometra sp. were found in four wild boar (8). All animals ( 1 female, 3 males) were hunted in BPF, Forestry District Browsk and Narewka, northeastern Poland. Twelve plerocercoid specimens were collected. They were found subcutaneously and in muscle tissue. The average length of sparganum was $103 \mathrm{~mm}$ (range 30-290 mm) (Fig. 4). The same method of molecular Spirometra sp. identification was used $(9,12)$. The sequences of $18 \mathrm{~S}$ rRNA gene fragment obtained from plerocercoids isolated from wild boar were published by Kołodziej-Sobocińska et al. (8). The sequences were identical to: the GeneBank - deposited Spirometra erinaceieuropaei sequence (D64072.1) (8), Spirometra sp. described previously in badger (9), and obtained from lynx (Kołodziej-Sobocińska et al., unpublished data). In addition, the phylogram based on 18S rRNA gene sequences extracted from the wild boar and badger from BPF, and compared with sequences accessed from GenBank resources, showed a very close relation between plerocercoids obtained from both hosts from BPF (8). As far as it is known, wild boar as a host of Spirometra sp. has never been reported in European Union countries and North America. It is most likely that the scavenger behavior as well as consumption of amphibians infected with Spirometra sp. plerocercoids resulted in wild boar infection. Additionally, the possibility of infection by drinking water with copepods Cyclops sp. infected with first stage larvae-procercoids cannot be ruled out.

\section{Human sparganosis}

Cases of human sparganosis have been well documented in Asia $(13,26)$. Most of them have been diagnosed as subcutaneous sparganosis $(13,25,26)$. However, pulmonary (3), eye anterior chamber (16) and cerebral (7) human sparganosis have also been documented. The only curative treatment is surgical extraction of the worm. All cases were connected with eating raw or undercooked meat of amphibians and reptiles $(13,26)$ or smoked wild boar meat $(25)$ in the medical history of the patients. Thus, the consump- tion of wild boar meat as a possible cause of human sparganosis cannot be excluded (25), as wild boar may be a paratenic host of Spirometra sp. as it has been recorded in Asia (11), Belarus (18) and recently in Poland (8).

\section{Diagnosis and prevention}

There are no sparganosis routine guidelines for veterinarians and there is a high probability that hunted wild boar infected with Spirometra sp. plerocercoids are not diagnosed and subsequently consumed by humans worldwide. Spargana are infective only a few days after developing from procercoids when only $1 \mathrm{~mm}$ in length (2), which significantly impedes the detection of this parasite in the consumed meat. Cases of probable sparganosis in wild boar have been noted in BPF since 2011, but no diagnostics have been performed until now (Miniuk, pers. commun.). In addition, wild boar meat can be infected with mesocercarial stage of the trematode Alaria alata (4). These both parasites can cause serious diseases in humans: sparganosis and alariasis. Knowing that in Poland alone 340,945 wild boar were hunted in 2015/2016 and the quantity of hunted wild boar procurement reached 5,096 tonnes (Central Statistical Office, Forestry 2016, Poland) attention should be paid to extend diagnostics of wild boar meat to detect these parasites. Of equal importance is dissemination of information on sparganosis effects and appropriate meat cooking procedures among both hunters and consumers, especially in the areas of high wild boar meat consumption.

The possibility of human sparganosis occurrence resulting from consumption of undercooked or smoked wild boar meat is very likely especially in the areas where wild boar is an approved food source. Public awareness of emerging zoonoses has increased considerably, as they potentially have a serious impact on human health and economy. It has therefore become a priority to inform the public about the possibilities and consequences of Spirometra sp. infection given the current situation. We suggest that extended guidelines are prepared for veterinarians on wild boar meat examination procedures, which would take into consideration the above findings and reports.

\section{References}

1. Anisimova E. I.: Study of the European mink Mustela lutreola helminthocenoses in connection with the American mink M. vison expansion in Belarus: story of the study and review of the results. Helminthologia 2004, 41, 193-196.

2. Bengston S. D., Rogers F.: Prevalence of sparganosis by county of origin in florida feral swine. Vet. Parasitol. 2001, 97, 239-242.

3. Cheng K. B., Gao B. L., Liu J. M., Xu J. F.: Pulmonary sparganosis mansoni: a case report from a non-endemic region. J. Thorac. Dis. 2014, 6, E120-1244.

4. Chmurzyńska E., Różycki M., Biska-Zając E., Karamon J., Cencek T.: Alaria alata - potential threat for humans, prevalence and diagnostic measures. Życie Wet. 2013, 88, 780-784.

5. Cornell S. J., Bjornstad O. N., Cattadori I. M., Boag B., Hudson P. J.: Seasonality, cohort-dependence and the development of immunity in a natural host-nematode system. P. Roy. Soc. B-Biol. Sci. 2008, 275, 511-518.

6. Furmaga S.: Spirometra janickii sp. n. (Diphyllobothriidae). Acta Parasitol. Polonica 1953, 1, 29-59. 
7. Jeong S. C., Bae J. C., Hwang S. H., Kim H. C., Lee B. C.: Cerebral sparganosis with intracerebral hemorrhage: A case report. Neurology 1998, 50, 503-506.

8. Kołodziej-Sobocińska M., Miniuk M., Ruczyńska I., Tokarska M.: Sparganosis in wild boar (Sus scrofa) - Implications for veterinarians, hunters, and consumers. Vet. Parasitol. 2016, 227, 115-117.

9. Kołodziej-Sobocińska M., Tokarska M., Kowalczyk R.: The first report of sparganosis (Spirometra sp.) in Eurasian badger (Meles meles). Parasitol. Int. 2014b, 63, 397-399.

10. Kołodziej-Sobocińska M., Zalewski A., Kowalczyk R.: Sarcoptic mange vulnerability in carnivores of the Białowieża Primeval Forest, Poland: underlying determinant factors. Ecol. Res. 2014a, 29, 237-244.

11. Lee S.-H., Choe E.-Y., Shin H.-D., Seo M.: Spargana in a weasel, Mustela sibirica manchurica, and a wild boar, Sus scrofa, from Gangwon-do, Korea. Korean J. Parasitol. 2013, 51, 379-381.

12. Lee S.-U., Chun H.-C., Huh S.: Molecular phylogeny of parasitic Platyhelminthes based on sequences of partial 28S rDNAD1 and mitochondrial cytochrome c oxidase subunit I. Korean J. Parasitol. 2007, 45, 181-189.

13. Liu Q., Li M. W., Wang Z. D., Zhao G. H., Zhu X. Q.: Human sparganosis, a neglected food borne zoonosis. Lancet Infect. Dis. 2015, 15, 1226-1235.

14. Manson P., Manson-Bahr P., Wilcocks C.: Manson's Tropical Diseases: A Manual of the Diseases. William Wood and Company, New York 1921.

15. Moller A. P.: Parasitism and the regulation of host populations. Parasitism Ecosystems 2005, 43-53

16. Mougeot G., Cambon M., Menerath J. M., Dimeglio V., Houin R.: Human eye anterior chamber sparganosis. Parasite-J. Soc. Fr. Parasitol. 1999, 6, 365-366.

17. Pampiglione S., Fioravanti M. L., Rivasi F.: Human sparganosis in Italy. Case report and review of the European cases. APMIS 2003, 111, 349-354.

18. Shimalov $V$. V.: Spirometrosis and sparganosis and other diphyllobothriases in the Republic of Belarus and their medical significance. Med. Parazitol. (Mosk) 2009, 48-52. (in Russian)
19. Shimalov $V$. V., Shimalov V. T.: Helminth fauna of the stoat (Mustela erminea Linneus, 1758) and the weasel (M. nivalis Linnaeus, 1758) in Belarusian Polesie. Parasitol. Res. 2001, 87, 680-681.

20. Shimalov V. V., Shimalov V. T.: Helminth fauna of the raccoon dog (Nyctereutes procyonoides Gray, 1834) in Belorussian Polesie. Parasitol. Res. 2002, 88, 944-945.

21. Shimalov V. V., Shimalov V. T., Shimalov A. V.: Helminth fauna of otter (Lutra lutra Linnaeus, 1758) in Belorussian Polesie. Parasitol. Res. 2000, 86, 528.

22. Sidorovich V., Bychkova E. I.: Helminth infestation in a declining population of European mink (Mustela lutreola) in Belarus. Small Carnivore Conservation 1993, 9, 16-17.

23. Solomon N. U., James I. M., Alphonsus N. O., Nkiruka R. U.: A review of host-parasite relationships. Ann. Res.Rev. Biol. 2015, 5, 372-385.

24. Szczęsna J., Popiołek M., Schmidt K., Kowalczyk R.: Coprological study on helminth fauna in Eurasian lynx (Lynx lynx) from the Białowieża Primeval Forest in Eastern Poland. J. Parasitol. 2008, 94, 981-984.

25. Tappe D., Berger L., Haeupler A., Muntau B., Racz P., Harder Y.: Case Report: Molecular diagnosis of subcutaneous Spirometra erinaceieuropaei sparganosis in a Japanese immigrant. Am. J. Trop. Med. Hyg. 2013, 88,198-202.

26. Wiwanitkit V.: A review of human sparganosis in Thailand. Int. J. Infect. Dis 2005, 9, 312-316.

27. Wongkulab P., Sukontason K., Chaiwarith R.: Sparganosis: A Brief Review. J. Infect. Dis. Antimicrob. Agents 2011, 28, 77-80.

28. Woolhouse M. E. J.: Patterns in parasite epidemiology: The peak shift. Parasitol. Today 1998, 14, 428-434.

29.Zuk M., McKean K. A.: Sex differences in parasite infections: Patterns and processes. Int. J. Parasitol. 1996, 26, 1009-1023.

Corresponding author: Marta Kołodziej-Sobocińska, PhD, Waszkiewicza 1, 17-230 Białowieża, Poland; e-mail: mksobocinska@ibs.bialowieza.pl 